\title{
TYPES OF $(2,2)$ POINT CORRESPONDENCES BETWEEN TWO
}

\section{PLANES*}

BY

\section{F. R. SHARPE AND VIRGIL SNYDER}

1. Introduction. The purpose of this paper is to obtain a classification of the possible $(2,2)$ point correspondences between two planes, and to describe the important features of each type. Consider two equations each algebraic in two sets of homogeneous coördinates $x_{1}, x_{2}, x_{3} ; x_{1}^{\prime}, x_{2}^{\prime}, x_{3}^{\prime}$. When $x_{1}, x_{2}, x_{3}$ are given, the equations are to represent two curves in the $\left(x^{\prime}\right)$ plane whose two variable intersections, that is, those depending on the parameters $x_{1}, x_{2}$, $x_{3}$, are the images of the point $(x) \equiv\left(x_{1}, x_{2}, x_{3}\right)$. Similarly if $x_{1}^{\prime}, x_{2}^{\prime}, x_{3}^{\prime}$ are given $x_{1}, x_{2}, x_{3}$ have two sets of values. By considering the possible forms of these equations, eleven independent types are obtained. Let $P_{1}$ have the images $P_{1}^{\prime}, P_{2}^{\prime}$; the images of $P_{1}^{\prime}$ are $P_{1}$ and $P_{2}$; the images of $P_{2}^{\prime}$ are $P_{1}$ and $\bar{P}_{2}$. Two distinct cases appear: When $P_{2}$ and $\bar{P}_{2}$ are distinct except for restricted positions of $P_{1}$, the correspondence will be said to belong to the general case. In this category belong Types $\mathrm{I}$ to $\mathrm{V}$. If $P_{2}$ is identical with $\bar{P}_{2}$ for every position of $P_{1}$, the correspondence will be called a compound involution. In this category belong Types VI to XI which are developed independently of Types I-V.

Finally it is shown that any $(2,2)$ point correspondence between two planes is birationally equivalent to one of the types here enumerated.

Only very special cases of $(2,2)$ correspondences have heretofore been studied, and practically none by the methods here employed. $\dagger$

2. The general case. If the two images of a point which is on the plane ( $x$ ) coincide, the point is on the curve of branch points. This curve will be designated by the symbol $L(x)$. The locus of the corresponding coincidences is a curve $K^{\prime}\left(x^{\prime}\right)$ which is in $(1,1)$ correspondence with $L(x)$. Similarly there is a curve of branch-points $L^{\prime}\left(x^{\prime}\right)$, and a curve of coincidences

* Presented to the Society, September 4, 1916.

† See Pascal's (German) Repertorium der höheren Mathematik, 2d edition, vol. 2, p. 371. All the results previously obtained appear as particular cases in our classification. No use is made of any of the papers there cited. See also R. Baldus, Zur Theorie der gegenseitig mehrdeutigen algebraischen Ebenentransformationen, $\mathrm{M}$ at he matis $\mathrm{ch}$ e A n $\mathrm{n}$ ale $\mathrm{n}$, vol. 72 (1912), pp. $1-36$. 
$K(x)$. The image of $L(x)$ is $K^{\prime}\left(x^{\prime}\right)$ counted twice. The image of $K^{\prime}\left(x^{\prime}\right)$ is $L(x)$ and a residual curve $R(x)$. We now require and shall frequently have occasion to make use of the following lemma.

Lemma. Let a point $P$ in $(x)$ describe a curve $C$. The necessary and sufficient condition that its images $P_{1}^{\prime}, P_{2}^{\prime}$, describe distinct curves is that $C$ touches $L$ at every common point. This follows immediately from the fact that $P_{1}^{\prime}$ and $P_{2}^{\prime}$ cannot interchange. Applying the lemma to the curves $K(x)$ and $K^{\prime}\left(x^{\prime}\right)$ we have the theorem.

Theorem. The curves $L(x), K(x)$ and $L^{\prime}\left(x^{\prime}\right), K^{\prime}\left(x^{\prime}\right)$ are tangent to each other at their common points.

If a line $c_{1}$ in $(x)$ meets $K(x)$ in $k$ points its image in $\left(x^{\prime}\right)$ is a curve $c^{\prime}$ tangent to $L^{\prime}\left(x^{\prime}\right)$ at $k$ points. If $c^{\prime}$ has $d$ variable double points, its image in $(x)$ is $c_{1}$ counted twice and a curve meeting $c_{1}$ in $k$ points corresponding to the $k$ contacts of $c^{\prime}$ with $L^{\prime}\left(x^{\prime}\right)$ and in $2 d$ points corresponding to the $d$ variable double points of $c^{\prime}$.

\section{NON-INVOLUTORIAL TYPES}

3. Types of correspondences. An algebraic correspondence between the points of the planes $(x)$ and $\left(x^{\prime}\right)$ may be expressed by two algebraic equations of the form

$$
\begin{aligned}
& \sum a_{i}\left(x^{\prime}\right) u_{i}(x)=0, \\
& \sum b_{i}\left(x^{\prime}\right) v_{i}(x)=0,
\end{aligned}
$$

where $a_{i}\left(x^{\prime}\right)=0, b_{i}\left(x^{\prime}\right)=0$ belong to linear systems of curves in the $\left(x^{\prime}\right)$ plane, and similarly for $u_{i}(x)=0, v_{i}(x)=0$ in the $(x)$ plane. The equations (1) and (2) define in each plane two algebraic systems of curves, the coördinates of a point in the other plane being the parameters. In each plane any curve of one system meets a curve of the other system in two variable points.

The following five cases give independent types but special cases of one type may sometimes be included in one or more of the remaining types.

$\begin{array}{rlll}\text { No. } a_{i}\left(x^{\prime}\right)=0 & u_{i}(x)=0 & b_{i}\left(x^{\prime}\right)=0 & v_{\boldsymbol{i}}(x)=0 \\ \text { I. conic } & \text { line } & \text { line } & \text { conic } \\ \text { II. conic } & \text { conic } & \text { line } & \text { line } \\ \text { III. conic } & \text { line } & \text { conic } & \text { conic } \\ \text { IV. conic } & \text { conic } & \text { conic } & \text { conic } \\ \text { V. } c_{n} & c_{n} & \text { line pencil } & \text { line pencil }\end{array}$

where $c_{n}$ is a curve of order $n$ with the basis point of the corresponding line pencil $(n-2)$-fold.

We proceed to discuss briefly each of these cases.

4. Type I. Image of a line. We may write the defining equations in the 
form

$$
\begin{aligned}
& \sum b_{i}(x) x_{i}^{\prime} \equiv \sum a_{i k}^{\prime}\left(x^{\prime}\right) x_{i} \cdot x_{k}=0, \\
& \sum b_{i}^{\prime}\left(x^{\prime}\right) x_{i} \equiv \sum a_{i k}(x) x_{i}^{\prime} \cdot x_{k}^{\prime}=0,
\end{aligned}
$$

wherein $a_{i k}, a_{i k}^{\prime}$ are linear, $b_{i}, b_{i}^{\prime}$ quadratic in the respective variables.

The image of a line

$$
\sum k_{i} x_{i}=0
$$

is the quintic

$$
\sum a_{i k}^{\prime}\left(x^{\prime}\right) X_{i}^{\prime} X_{k}^{\prime}=0
$$

wherein $X_{i}^{\prime}$ is the cofactor of $x_{i}^{\prime}$ in the determinant

$$
\left|\begin{array}{lll}
x_{1}^{\prime} & x_{2}^{\prime} & x_{3}^{\prime} \\
b_{1}^{\prime} & b_{2}^{\prime} & b_{3}^{\prime} \\
k_{1} & k_{2} & k_{3}
\end{array}\right| \text {. }
$$

The quintic (4) is of genus 2 , having double points at the four points given by

$$
\frac{b_{1}^{\prime}}{k_{1}}=\frac{b_{2}^{\prime}}{k_{2}}=\frac{b_{3}^{\prime}}{k_{3}} \text {. }
$$

5. Curves of branch points and of coincidences. The two images of a point of $\left(x^{\prime}\right)$ coincide if the line $\left(2^{\prime}\right)$ touches the conic $\left(1^{\prime}\right)$. The curve of branch points in $\left(x^{\prime}\right)$ is therefore the sextic of genus 10

$$
L^{\prime}\left(x^{\prime}\right) \equiv\left|\begin{array}{llll}
a_{11}^{\prime} & a_{12}^{\prime} & a_{13}^{\prime} & b_{1}^{\prime} \\
a_{21}^{\prime} & a_{22}^{\prime} & a_{23}^{\prime} & b_{3}^{\prime} \\
a_{31}^{\prime} & a_{32}^{\prime} & a_{33}^{\prime} & b_{3}^{\prime} \\
b_{1}^{\prime} & b_{2}^{\prime} & b_{3}^{\prime} & 0
\end{array}\right|=0 .
$$

The curve of coincidences $K^{\prime}\left(x^{\prime}\right)$ counted twice is the image of $L(x)$, which is similar to $L^{\prime}\left(x^{\prime}\right)$. It is of order 15 and genus 10 , and has 45 contacts with $L^{\prime}\left(x^{\prime}\right)$.

6. Locus of double points in the images of a pencil of lines. The images of the point $(0,0,1)$ in $(x)$ lie on the conic $b_{3}^{\prime}=0$. From (5) this conic also passes through the four double points of the quintic corresponding to any lire through $(0,0,1)$. Hence the theorem:

Theorem. The two images of a point and the double points of the quintic images of the pencil of lines through the point lie on a conic.

7. Successive images of a line. It has just been shown that the image of a line $c_{1}$ in $(x)$ is a quintic $c_{5}^{\prime}$ having four double points and 15 contacts with $L_{6}^{\prime}$ which correspond to the 15 intersections of $c_{1}$ with $K_{15}$. The image of $c_{5}^{\prime}$ is therefore $c_{1}$ counted twice and a residual $c_{23}$ of genus 2 passing through the 15 intersections of $c_{1}$ with $K_{15}$ and through 8 other points on $c_{1}$, images of the 4 double points of $c_{5}^{\prime}$. The images of two lines $c_{1}, \bar{c}_{1}$ are two quintics 
$c_{5}^{\prime}, \bar{c}_{5}^{\prime}$ which meet in 25 points. Two of these points correspond to the common point of $c_{1}, \bar{c}_{1}$. The remaining 23 have for images the 23 intersections of $c_{1}$ with $\bar{c}_{23}$ and $\bar{c}_{1}$ with $c_{23}$. Since the curve $c_{23}$ has a composite image it has 69 contacts with $L_{6}$. The 75 intersections of $c_{5}^{\prime}$ with $K_{15}^{\prime}$ are the images of these 69 points and of the 6 intersections of $c_{1}$ with $L_{6}$. The image of $c_{23}$ is $c_{5}^{\prime}$ and a residual $c_{110}^{\prime}$ passing through the 4 double points of $c_{5}^{\prime}$ and the 15 contacts of $c_{5}^{\prime}$ with $L_{6}^{\prime}$. The curve $c_{110}^{\prime}$ has 330 contacts with $L_{6}^{\prime}$ which correspond to the 330 points in which $c_{23}$ meets $K_{15}$ apart from the 15 which lie on $c_{1}$. The curves $c_{5}^{\prime}, c_{110}^{\prime}$ meet in 550 points, namely 8 for the 4 double points of $c_{5}^{\prime}, 15$ for the points of contact of $c_{5}^{\prime}$ with $L_{6}^{\prime}, 69$ on $K_{15}^{\prime}$, images of the 69 contacts of $c_{23}$ and $L_{6}$, and finally 458 images of the 229 double points of $c_{23}$. By carrying the process one step further we can see the general law. The image of $c_{110}^{\prime}$ is $c_{23}$ and a residual $c_{527}$ which meet in 12121 points, made up as follows: the 23 intersections of $c_{1}, c_{23} ; 330$ points of $c_{23}$ on $K_{15}$ but not on $c_{1} ; 5884$ pairs of points, images of the double points of $c_{110}^{\prime}$. The curve $c_{527}$ does not pass through the double points of $c_{23}$, although $c_{110}^{\prime}$ passes through the double points of $c_{5}^{\prime}$. The reason of this is that both images of a point of $c_{1}$ are on $c_{5}^{\prime}$, while $c_{23}$ and $c_{527}$ are in $(1,1)$ correspondence.

8. Case of conics in $\left(x^{\prime}\right)$ with one basis point. Let the basis point be taken as $(1,0,0) \equiv A^{\prime}$. The image of $c_{1}$ is $c_{5}^{\prime}$ with one double point at $A^{\prime}$ and three variable double points. The curve $L_{6}^{\prime}$ has a double point at $A^{\prime}$ and is of genus 9. For points in the neighborhood of $A^{\prime}$ we have

$$
b_{1}=0, \quad a_{12} x_{2}^{\prime}+a_{13} x_{3}^{\prime}=0,
$$

hence to each direction through $A^{\prime}$ correspond 2 points on the conic $b_{1}=0$ which is the image of $A^{\prime}$. The image of $c_{5}^{\prime}$ is $c_{1}^{2}$, the conic $b_{1}$ counted twice and a residual $c_{19}$ of genus 2 . The image of $L_{6}^{\prime}$ is $b_{1}$ and $K_{13}$ of genus 9 . The curve $c_{19}$ meets $c_{1}$ in 13 points on $K_{13}$ and in the 6 points-images of the three variable double points of $c_{5}^{\prime}$. The conic $b_{1}$ touches $L_{6}$ in 6 points; hence $K_{15}^{\prime}$ has $A^{\prime}$ sixfold and 66 double points. The image of $K_{15}^{\prime}$ is $L_{63}\left(b_{1}\right)^{6}$ and a residual $C_{57}$ touching $L_{6}$ in the 39 points of contact with $K_{13}$ and in the 132 point-images of the 66 double points of $K_{15}^{\prime}$. The image of $b_{1}$ is the point $A^{\prime}$ and a rational $C_{10}^{\prime}$ having $A^{\prime}$ sixfold. The curves $L_{6}^{\prime}, c_{10}^{\prime}$, meet in 60 points, namely 12 at $A_{1}$, two contacts, images of the contacts of $b_{1}$ and $K_{13}$, since the two images on $b_{1}$ of a tangent to $L_{6}^{\prime}$ coincide, and in 22 other contacts, images of the 22 remaining intersections of $b_{1}$ and $K_{13}$.

A line in $\left(x^{\prime}\right)$ through $A^{\prime}$ has for image the conic $b_{1}$ and a cubic curve of genus 1. Two lines of the pencil $A^{\prime}$ have no other point in common, hence the nine points of intersection of their image cubics must be accounted for. The image of $c_{3}$ is a curve of order 15 having $A^{\prime}$ for a sixfold point; but $c_{1}^{\prime}$ appears twice as a component, hence the residual is a $c_{13}^{\prime}$, having $A$ for a 
fourfold point. The curve $c_{13}^{\prime}$ meets $c_{1}^{\prime}$ in 9 points besides $A$; they are at the nine points in which $c_{1}^{\prime}$ meets $K_{15}^{\prime}$ besides $A$, which was seen to be a sixfold point on $K_{15}^{\prime}$.

Now consider two lines $c_{1}^{\prime}, \bar{c}_{1}^{\prime}$; their images $c_{3}, \bar{c}_{3}$ meet in 9 points, whose images in $\left(x^{\prime}\right)$ lie 9 on $c_{1}^{\prime}, \bar{c}_{13}^{\prime}$ and 9 on $\bar{c}_{1}^{\prime}, c_{13}^{\prime}$. Two of the intersections of $c_{3}$ with $b_{1}$ are images of the direction of $c_{1}^{\prime}$ through $A$.

A curve of order $n$ in $(x)$ meets $b_{1}$ in $2 n$ points; if these points are all independent, so that no two of them correspond to the same direction through $A$, the image curve in $\left(x^{\prime}\right)$ will have a $2 n$-fold point at $A$. But if the curve goes through a pair of conjugate points both points indicate but a single tangent to the image curve at $A$. A third alternative is illustrated by the images of an arbitrary straight line. Let $c_{1}$ meet $b_{1}$ in $P_{1}, P_{2}$. The images of $P_{1}, P_{2}$ are the two tangents $t_{1}, t_{2}$ to $c_{5}^{\prime}$ at $A$, each of which has another image $\bar{P}_{1}, \bar{P}_{2}$ on $b_{1}$. The residual image of $c_{5}^{\prime}$ is $c_{19}$, which meets $b_{1}$ in 38 points. But two of these points are $\bar{P}_{1}, \bar{P}_{2}$. The two images of $\bar{P}_{1}$ are $t_{1}$ and a point in $\left(x^{\prime}\right)$ on the residual image of $c_{19}$; similarly for $\bar{P}_{2}$. Hence the residual image of $c_{19}$ has $A$ for a multiple point of order 36 . This alternative is possible only when the image of the curve under consideration is composite.

9. Case of conics in $(x)$ and $\left(x^{\prime}\right)$ with one basis point. Let the basis points be $A$ and $A^{\prime}$ each $(1,0,0)$ in $(x)$ and $\left(x^{\prime}\right)$. The image of $A$ is $b_{1}^{\prime}=0$ through $A^{\prime}$, and of $A^{\prime}$ is $b_{1}=0$ through $A$. Hence we have the following theorem.

THEOREM. If the conics in each plane have a basis point, each basis point lies on the image conic of the other.

The details of this case are in other respects similar to the preceding case.

10. Case of conics in $\left(x^{\prime}\right)$ having two basis points. Let the basis points be $A^{\prime} \equiv(1,0,0)$ and $B^{\prime} \equiv(0,1,0)$. For points on the line $A^{\prime} B^{\prime}$ we have $b_{1} x_{1}^{\prime}+b_{2} x_{2}^{\prime}=0, a_{12}=0$. Hence to a point on $A^{\prime} B^{\prime}$ correspond two points on the line $a_{12}=0$. The image of the line $A^{\prime} B^{\prime}$ is $b_{1}, b_{2}$, and $a_{12}$.

The image of $a_{12}$ is $A^{\prime} B^{\prime}$ taken twice, and a rational $c_{3}^{\prime}$ not passing through $A^{\prime}$ or $B^{\prime}$. The line $a_{12}$ is a tri-tangent to $L_{6}$, hence $A^{\prime} B^{\prime}$ meets $c_{3}^{\prime}$ in three points on $K_{15}^{\prime}$. Since $K_{15}^{\prime}$ has $A^{\prime}$ and $B^{\prime}$ for sixfold points, $A^{\prime} B^{\prime}$ has no other points on $K_{15}^{\prime}$.

The line $A^{\prime} B^{\prime}$ meets $L_{6}^{\prime}$ in two points besides $A^{\prime}, B^{\prime}$. The images of these points are the points of contact of the conics of the pencil $b_{1}, b_{2}$ which touch $a_{12}$.

The cases of two basis points in one plane, together with one or two in the other plane present no difficulties.

11. Three basis points in $\left(x^{\prime}\right)$, none in $(x)$. Since they can not all be collinear, we may use the triangle having the basis points for vertices as triangle of quadratic inversion and reduce the conics of the system through them to straight lines, and the straight lines expressed in the other equation to conics, 
thus reducing the defining equations to a particular case of Type II. Similar transformations can be made when the lines have a basis point. They can be transformed into a pencil of conics whether the coefficients are linear or quadratic. In either case the curves defined by the same equation in the other plane also belong to a pencil.

12. Other particular forms of Type I. Suppose $x_{1}=0, x_{1}^{\prime}=0$ satisfy both equations. The image of $c_{1}$ has $x_{1}^{\prime}=0$ as a fixed component, the variable component being a quartic of genus 1 . The curve $L_{6}$ has $x_{1}^{2}=0$ as a component, the residual curve being a quartic of genus 1 .

If $x_{2}=0, x_{2}^{\prime}=0$ also satisfy both equations, there are three basis points common to the conics in each plane. The proper image of a straight line is now a rational cubic. The curve $L_{2}$ is a conic and $K_{3}$ a rational cubic.* If finally both equations are also satisfied by $x_{3}=0, x_{3}^{\prime}=0$ they may be written in the form

$$
\begin{aligned}
x_{1}^{\prime} x_{2} x_{3}+x_{2}^{\prime} x_{3} x_{1}+x_{3}^{\prime} x_{1} x_{2}=0, \\
a_{1} x_{1} x_{2}^{\prime} x_{3}^{\prime}+a_{2} x_{2} x_{3}^{\prime} x_{1}^{\prime}+a_{3} x_{3} x_{1}^{\prime} x_{2}^{\prime}=0 .
\end{aligned}
$$

The fundamental points in each plane are

$$
(1,0,0), \quad(0,1,0), \quad(0,0,1),
$$

and the associated fundamental conics are

$$
x_{2} x_{3}=0, \quad x_{3} x_{1}=0, \quad x_{1} x_{2}=0 .
$$

Each fundamental point is a double point on a fundamental conic. The equations define two collineations of the form $x_{i}=k_{i} x_{i}^{\prime}$ which can be rationally separated.

Both $L$ and $K$ vanish identically.

13. Type II. A straight line in either plane has for image a quartic with one variable double point. The curve $L$ is of order 6 and genus $10 ; K$ is of order 12. These two curves have 36 points of contact. For every basis point of the conics in either plane $L_{6}$ has a double point, and in the other plane there is a fundamental line. The extreme particularization

$$
b_{1}(x) x_{1}^{\prime}+b_{2}(x) x_{2}^{\prime}=0, \quad b_{1}^{\prime}\left(x^{\prime}\right) x_{1}+b_{2}^{\prime}\left(x^{\prime}\right) x_{2}=0
$$

having five basis points in each plane, with four fundamental conics belonging to a pencil, and a fundamental line, is a direct generalization of the Seydewitz $\dagger$ method of defining a birational quadratic inversion. All the properties of

* This is one of the cases treated by Marletta, Rendicon ti del Circolo M a t ematico di Paler mo, vol. 17 (1903), pp. 173-184, 371-385. The lines $x_{1}=0, x_{1}^{\prime}=0$ are illustrations of the curves D defined by Baildus, l. c. The curves D appear only in subcases of more general types in our classification.

†A A chivder Mathematik und Physik, vol. 7 (1846), pp. 113-148. 
this transformation can be derived readily by the methods given in the present paper.

14. Particular cases of Type II. If both equations are satisfied by $x_{1}=0$, $x_{1}^{\prime}=0$ the image of a line is a cubic curve of genus 1 . The curve $L$ is a quartic of genus 3 and $K$ a sextic. If $x_{2}=0, x_{2}^{\prime}=0$ also satisfy both equations the image of a line is a conic with no basis points. The curves $L$ and $K$ are both conics having double contact with each other.*

15. Type III. Consider the conics in $(x)$ through $B \equiv(0,1,0)$ and $C \equiv(0,0,1)$

$$
\begin{gathered}
a x_{1}^{2}+b x_{1} x_{2}+c x_{1} x_{3}+d x_{2} x_{3}=0, \\
p x_{1}^{2}+q x_{1} x_{2}+r x_{1} x_{3}+s x_{2} x_{3}=0,
\end{gathered}
$$

where $a, b, c, d$ are quadratic and $p, q, r, s$ linear in $\left(x^{\prime}\right)$. Proceeding as in Type I the image of $c_{1}^{\prime}$ is $c_{6}$, a sextic with triple points at $B$ and $C$ and two variable double points. $L$ is of order 8 and genus 9 having fourfold points at $B$ and $C$. To find the image of a point near $B$ we have

$$
b x_{1}+d x_{3}=0, \quad q x_{1}+s x_{3}=0 .
$$

Hence to a direction through $B$ correspond 2 points on the cubic $b s-d q=0$, which is the image of $B$. Similarly for $C$. Corresponding to any point on $B C$ are the two points $B^{\prime} C^{\prime}$ given by $d=s=0$. To find the image of a line $c_{1} \equiv \Sigma k_{i} x_{i}=0$, replace the first of the defining equations by

$$
(a s-p d) x_{1}+(b s-q d) x_{2}+(c s-r d) x_{3}=0 .
$$

Hence proceeding as in Type I, the image of a line is a curve of order 7 with double points at the 9 points given by

$$
\frac{a s-p d}{k_{1}}=\frac{b s-q d}{k_{2}}=\frac{c s-r d}{k_{3}},
$$

of which 2 are at $B^{\prime}$ and $C^{\prime}$ and 7 variable. But $s=0$ is a fixed component so that the image of $c_{1}$ is $c_{6}^{\prime}$ having 7 double points and passing through $B$ and $C$. Similarly $L^{\prime}$ is of order 6 because the curve of order 8 obtained contains $s=0$ twice as an extraneous factor. The curve $K$ is of order 18 , with $B$ and $C 9$-fold points. The curve $K^{\prime}$ is of order 12 .

16. Type IV. If in the equations used for Type III the coefficients of the $(x)$ 's are all quadratic in the $\left(x^{\prime}\right)$ 's and have no terms in $x_{2}^{\prime 2}$ or ${x_{3}^{\prime 2}}^{2}$ there are 2 basis points $B^{\prime} \equiv(0,1,0)$ and $C^{\prime} \equiv(0,0,1)$. Proceeding as in Type III to replace one of the equations by

$$
(a s-p d) x_{1}+(b s-q d) x_{2}+(c s-r d) x_{3}=0,
$$

the image of a line

$$
c_{1} \equiv \sum k_{i} x_{i}=0
$$

* Both cases were discussed by Marletta, l. c., $\S 12$. 
is $c_{8}^{\prime}$ with $B^{\prime}$ and $C^{\prime}$ fourfold points, 6 variable double points and passing simply through $E^{\prime}$ and $F^{\prime}$, the residual intersections of $d=0, s=0$. The curve $L^{\prime}$ is of order 8 , with $B^{\prime}$ and $C^{\prime}$ fourfold points. For points near $B$, we have

$$
b x_{1}+d x_{3}=0, \quad q x_{1}+s x_{3}=0 .
$$

Hence to each direction through $B$ correspond two points on the quartic $b s-d q=0$, which has double points at $B^{\prime}$ and $C^{\prime}$, simple points at $E^{\prime}$ and $F^{\prime}$ and is the image of $B$. The image of a point on $B^{\prime} C^{\prime}$ is $E$ and $F$. The image of a general curve of order 8 in $\left(x^{\prime}\right)$ is of order 64 , with $B$ and $C$ for 32 -fold points, and $E$ and $F 8$-fold points. In the case of $L_{8}^{\prime}$ the quartic images of $B^{\prime}$ and $C^{\prime}$ are to be taken off each 4 times, leaving for $K^{2}$ a curve of order 32 , having $B$ and $C 16$-fold. Hence $K$ is of order 16 , with $B$ and $C$ each 8-fold. In the case of $c_{8}^{\prime}$ we must also take off $B C$ (the image of $E$ and $F$ ) twice, and also $c_{1}^{\prime}$. The residual is of order 28 with $B$ and $C$ 14-fold points.

17. Type V. The equations (1), (2) have the forms

$$
\sum b_{i} b_{i}^{\prime}=0, \quad x_{1} x_{1}^{\prime}+x_{2}^{\prime} x_{2}=0,
$$

wherein

$$
b_{i}=x_{3}^{2} \phi_{n-2, i}\left(x_{1}, x_{2}\right)+x_{3} \phi_{n-1, i}\left(x_{1}, x_{2}\right)+\phi_{n, i}\left(x_{1}, x_{2}\right)=0
$$

and $b_{i}^{\prime}$ is similar, but of order $n^{\prime}$.

The point $(0,0,1)$ in each plane is the only fundamental point. Its image is of order $n+n^{\prime}-2$ having $(0,0,1)\left(n+n^{\prime}-4\right)$-fold. The image of a line is a curve of order $n+n^{\prime}$ having $(0,0,1)\left(n+n^{\prime}-2\right)$-fold. The curves of branch points are of order $2 n+2 n^{\prime}-2$, having $(0,0,1)(2 n$ $\left.+2 n^{\prime}-6\right)$-fold. The curves of coincidences are of order $4 n+4 n^{\prime}-6$, having $(0,0,1)\left(4 n+4 n^{\prime}-10\right)$-fold.

\section{Compound Involutions}

18. Forms of the equations. In the compound involutions, a point $P_{1}$ has $P_{1}^{\prime}, P_{2}^{\prime}$ for images; the images in $(x)$ of $P_{1}^{\prime}$ are $P_{1}$ and $P_{2}$; those of $P_{2}^{\prime}$ are the same points $P_{1}$ and $P_{2}$. Hence, if $P_{1}$ or $P_{2}$ is given, the other is uniquely determined, that is, in each plane the two images of a point in the other belong to a simple involution. This fact enables us to map the plane $(x)$ on a double plane $(y)$ by means of a $(1,2)$ transformation, and similarly the plane $\left(x^{\prime}\right)$ on a double plane $\left(y^{\prime}\right)$. The planes $(y)$ and $\left(y^{\prime}\right)$ must be birationally equivalent. An involution can always be defined by a net of curves, and mapped on a double plane $(y)$ by equations of the form

$$
\frac{\phi_{1}(x)}{y_{1}}=\frac{\phi_{2}(x)}{y_{2}}=\frac{\phi_{3}(x)}{y_{3}},
$$


where $\phi_{i}=0$ define a net of curves with two intersections that are functions of $y_{1}, y_{2}, y_{3}$. The second transformation is

$$
y_{i}^{\prime}=\psi_{i}(y),
$$

a Cremona transformation, and the third is

$$
\frac{\phi_{1}^{\prime}\left(x^{\prime}\right)}{y_{1}^{\prime}}=\frac{\phi_{2}^{\prime}\left(x^{\prime}\right)}{y_{2}^{\prime}}=\frac{\phi_{3}^{\prime}\left(x^{\prime}\right)}{y_{3}^{\prime}},
$$

wherein the curves define a net in $\left(x^{\prime}\right)$ with two intersections that are functions of $y_{1}^{\prime}, y_{2}^{\prime}, y_{3}^{\prime}$.

By eliminating $y_{1}, y_{2}, y_{3} ; y_{1}^{\prime}, y_{2}^{\prime}, y_{3}^{\prime}$ from these equations we have the following theorem.

TheOREM. The necessary and sufficient condition that a $(2,2)$ transformation is a compound involution is that the equation which define it may be reduced to the form

$$
\frac{F_{1}(x)}{F_{1}^{\prime}\left(x^{\prime}\right)}=\frac{F_{2}(x)}{F_{2}^{\prime}\left(x^{\prime}\right)}=\frac{F_{3}(x)}{F_{3}^{\prime}\left(x^{\prime}\right)},
$$

in which any two curves represented by these equations and lying in one plane meet in two variable points. In geometric form, the theorem states that the sufficient condition that a $(2,2)$ transformation is a compound involution is that the defining curves in one of the planes (and hence also in the other) compose a net.

Compound involutions appear as particular cases of each of the types already mentioned, but others exist which cannot be thus expressed.

19. Properties of compound involutions. Let $c$ be any curve in $(x)$. As in the preceding cases, the image of $c$ is a curve $c^{\prime}$, touching $L^{\prime}$ at every common point; but in the present case, the image of $c^{\prime}$ in $(x)$ consists of $c$ and of a residual curve $\bar{c}$, each counted twice. The curve $\bar{c}$ is not a contact curve of $L$, and $c^{\prime}$ is the complete image of $\bar{c}$.

When $P$ describes $L$, its images $P_{1}^{\prime} \equiv P_{2}^{\prime}$ describe $K^{\prime}$, and the residual image of $P_{1}^{\prime}$ also lies on $L$. Hence the image of $L$ is $K^{\prime}$ counted four times, and the complete image of $K^{\prime}$ is $L$. Hence $L, K^{\prime}$ are not in $(1,1)$ correspondence. Since the image of $K$ is not composite, $K$ is not a contact curve of $L$.

The curves $K, K^{\prime}$ are the jacobians of the nets in their respective planes.

In the following classification, two transformations are regarded as equivalent when their two component $(1,2)$ transformations are respectively equivalent. For simplicity it is here assumed that linear relations between $(y)$ and $\left(y^{\prime}\right)$ exist, such that the fundamental elements of tine two $(1,2)$ transformations are distinct.

20. The $(\mathbf{I}, 2)$ transformations. The $(1,2)$ plane transformations have 
been extensively studied.* They are of three types. The first is obtained by the intersections of a line of a plane field with an associated conic of a net, or of the cubics of a net through seven fixed points. It will be called the Geiser type. The second is given by the intersection of a line of a pencil 0 with a curve of order $n$ of a net having 0 for $(n-2)$-fold point. This will be called the Jonquières type. The third is found by the variable intersections of a cubic belonging to a pencil, with an associated sextic of a pencil having eight of the basis points of the cubics for double points. It will be called the Bertini type. By combining these various types we obtain the following Types of $(2,2)$ compound involutions.

VI. Geiser, Geiser.

VII. Geiser, Jonquières.

VIII. Geiser, Bertini.

IX. Jonquières, Jonquières.

$\mathrm{X}$. Jonquières, Bertini.

XI. Bertini, Bertini.

Before discussing these types it will be convenient to state the principal properties of the three component simple involutions.

The Geiser type. The image of a line $c_{1}(y)$ is a cubic with seven simple basis points $A_{i}$. The curve $K$ is a sextic, having each point $A_{i}$ double. The curve $L(y)$ is a quartic of genus 3 . There are no fundamental elements in $(y)$. A line $c_{1}(x)$ goes into a cubic curve with one variable double point and touching $L(y)$ in six points. The image of a point $A_{i}$ is a bitangent to $L(y)$.

The Jonquières type. A line $c_{1}(y)$ goes into $c_{n}(x)$ having $C_{x}(n-2)$-fold and $4 n-6$ simple basis points $B_{i}(x)$. The point $C_{y}$ goes into $C_{n-1}(x)$ having $C_{x}(n-3)$-fold and the points $B_{i}(x)$ simple. The curve $K(x)$ is of order $2 n-2$ having $C_{x}(2 n-4)$-fold and the points $B_{i}$ simple. A line $c_{1}(x)$ goes into $C_{n}(y)$ having $C_{y}(n-1)$-fold. The point $C_{x}$ goes into $C_{n-2}(y)$ having $C_{y}(n-3)$-fold. Each point $B_{i}$ goes into a line through $C_{y}$. The curve $L_{y}$ is of order $2 n-2$ having $C_{y}(2 n-4)$-fold.

The Bertini type. The line $c_{1}(y)$ goes into $c_{6}(x)$, having 8 double points $A_{i}$ and 2 simple basis points $D_{i}$. The curve $K(x)$ is of order nine having the points $A_{i}$ triple. The curve $L(y)$ is a sextic with two consecutive triple points at a point $E(y)$, the tangent being a line $\gamma$. The image of $E(y)$ is a cubic through the points $A_{i}$ and $D_{i}$. The image of a point $A_{i}$ is a conic touching $\gamma$ at $E(y)$ and touching $L(y)$ in three other points. The image of a point $D_{i}$ is the line $D_{y} E_{y} \equiv \gamma$. The line $c_{1}(x)$ goes into a sextic with two consecutive triple points at $E(y)$, touching $L(y)$ in nine points and having four variable double points.

\footnotetext{
* See Pascal's Repertorium, l. c., pp. 366-370, for the principal literature.
} 
21. Type VI. Each fundamental point $A_{i}$ goes intc an elliptic cubic of the net in $\left(x^{\prime}\right)$. A line $c_{1}$ goes into $c_{9}^{\prime}$ with seven triple points at $A_{i}^{\prime}$, and two variable double points.

$K_{6}$ goes into $L_{12}^{\prime}$ having $A_{i}^{\prime}$ for fourfold points. It is of genus 13 .

The image of $c_{9}^{\prime}$ is a curve of order 81 in $(x)$, consisting of seven fundamental cubics, images of $A_{i}^{\prime}$, each counted three times, the original $c_{1}$ counted twice, and the rational $c_{8}$ with triple points at $A_{i}$, image of $c_{1}$ in the Geiser involution in $(x)$, also counted twice. The line $c_{1}$ and the curve $c_{8}$ have no restricted position as to $L$, but $c_{9}^{\prime}$ touches $L^{\prime}$ in each common point. The curve $c_{9}$ is the complete image of $c_{8}$.

22. Type VII. The fundamental points $A_{i}$ in $(x)$ go into seven curves of a Jonquières net, of order $n$. The only fundamental points in $\left(x^{\prime}\right)$ are the point $C^{\prime}=(0,0,1),(n-2)$-fold on all curves of order $n$, and $4 n-6$ simple basis points $B_{i}^{\prime}$. The $(y)$ image of the multiple point is a curve of order $n-2$, with an $(n-3)$-fold point $C_{y}$, and its image in $(x)$ is of order $3(n-2)$, having seven points of order $n-2$ at $A_{i}$, and two points $C_{1}, C_{2}$, each of multiplicity $n-3$. The $(y)$ images of the simple points are $4 n-6$ lines of the pencil $C_{y}$; the $(x)$ images of these lines are $4 n-6$ cubics of the pencil through the seven basis points $A_{i}$ and $C_{1}, C_{2}$.

A line $c_{1}(x)$ goes into a nodal $c_{3}$ in $(y)$, which goes into $c_{3 n}^{\prime}$, having $C^{\prime}$ of multiplicity $3(n-2)$, and having $4 n-6$ threefold points at $B_{i}^{\prime}$. A line $c_{1}^{\prime}$ goes into $c_{n}(y)$ with $C_{y}$ for $(n-1)$-fold point; its image in $(x)$ is $c_{3 n}$ with $n$-fold points at $A_{i}$ and two $(n-1)$-fold points $C_{1}^{\prime}, C_{2}$. The curve $K_{6}$ goes into $c_{4}(y)$, whose image $L^{\prime}$ in $\left(x^{\prime}\right)$ is of order $4 n$, having $C^{\prime}$ as $4(n-2)$ fold point, and $4 n-6$ points $B_{1}^{\prime}$, each of multiplicity 4 . The curve $K_{2(n-1)}^{\prime}$ goes into $L_{2(n-1)}(y)$ with $C_{y}$ of multiplicity $2(n-2)$ and this goes into $L_{6(n-1)}$ with $A_{i}$ each of multiplicity $2(n-1)$ and two points $C_{1}, C_{2}$, each of multiplicity $2(n-2)$.

23. Type VIII. The fundamental points $A_{i}$ go into seven curves of order 6 belonging to the net in $\left(x^{\prime}\right)$. The points $E_{i}^{\prime}$ go into eight sextics having double points at $A_{i}$. The points $D_{i}$ go into a cubic of the net. A line $c_{1}$ goes into $c_{18}^{\prime}$ with sixfold points at $E_{i}^{\prime}$, two triple points at $D_{1}^{\prime}, D_{2}^{\prime}$, and two variable double points. The line $c_{1}^{\prime}$ goes into $c_{18}$ with sixfold points at $A_{i}$, two consecutive triple points at $F_{1}, F_{2}$, and eight variable double points.

The curve $L_{24}^{\prime}$ has eightfold points at $E_{i}^{\prime}$, and fourfold points at $D_{1}^{\prime}, D_{2}^{\prime}$, while $L_{18}$ has consecutive triple points at $F_{\mathrm{i}}, F_{2}$, and sixfold points at $A_{i}$.

The residual image of $c_{18}^{\prime}$ is $c_{17}^{\prime}$, image of $c_{1}$ in the Cremona invelution of Bertini type.

24. Type IX. A line $c_{1}$ goes into $c_{n}(y)$ with $C_{y}$ as $(n-1)$-fold point; this goes into $c_{n n^{\prime}}^{\prime}$, having $C^{\prime}$ as point of order $n\left(n^{\prime}-2\right)$, two points of multiplicity $n-1$, and having $4 n^{\prime}-6$ points, each of multiplicity $n$. The curve 
$K_{2 n-2}$ has $C$ of multiplicity $2 n-4$, and $4 n-6$ simple points $B_{i}$, hence $L_{2 n(n-1)}^{\prime}$ has $C^{\prime}$ of order $2(n-1)\left(n^{\prime}-2\right), 4 n^{\prime}-6$ points $B_{i}$ each of order $2(n-1)$, and two points of order $2 n-4$. By interchanging $n$ and $n^{\prime}$, we obtain $K^{\prime}$ and $L$.

25. Type $\mathrm{X}$. The points $E_{i}$ go into curves of order $2 n$, having $C^{\prime}$ as $2(n-2)$-fold point, $4 n-6$ points $B_{i}$ for double points. The point $C^{\prime}$ goes into a curve of order $6(n-2)$ having $E_{i}$ for $2(n-2)$-fold points, two $(n-3)$-fold points, and two $(n-2)$-fold points $D_{1}, D_{2}$. A line $c_{1}$ goes into $c_{6 n}^{\prime}$, having $C^{\prime}$ as $6(n-2)$-fold point, $B_{i}^{\prime}$ for sixfola points, two consecutive triple points at $F_{1}^{\prime}, F_{2}^{\prime}$, and eight variable double points.

A line $c_{1}^{\prime}$ goes into $c_{6 n}$, with two points $C_{1}, C_{2}$ of multiplicity $n-1$, the points $E_{i}$ of multiplicity $2 n$, and $D_{1}, D_{2} n$-fold. The curve $L$ is of order $12(n-1)$, has $E_{i}$ for points of multiplicity $4(n-1), D_{1}, D_{2}$ of multiplicity $2(n-1), C_{1}, C_{2}$ of multiplicity $2(n-2)$. The curve $L^{\prime}$ is of order $6 n$, has consecutive triple points at $F_{1}^{\prime}, F_{2}^{\prime}$ and $C^{\prime}$ for point of multiplicity $6(n-2), B_{i}^{\prime}$ of multiplicity 6 .

26. Type XI. The points $E_{i}$ go into curves of order 12 having $E_{i}^{\prime}$ for fourfold points, and $D_{1}^{\prime}, D_{2}^{\prime}$ for double points. The line $c_{1}$ goes into $c_{36}^{\prime}$ having 12-fold points at $E_{i}^{\prime}$, sixfold points at $D_{1}^{\prime}, D_{2}^{\prime}$, two consecutive triple points at $F_{1}^{\prime}, F_{2}^{\prime}$ and eight variable double points.

The curve $L_{36}^{\prime}$ has eight 12 -fold points at $E_{i}^{\prime}$, sixfold points at $D_{1}^{\prime}, D_{2}^{\prime}$, and two consecutive triple points at $F_{1}^{\prime}, F_{2}^{\prime}$.

27. Relations between the two lists. If the equations (1) and (2) each contain but two terms the resulting particular forms of Types $\mathrm{I}-\mathrm{V}$ can be expressed as follows:

$$
\begin{gathered}
u_{1}(x) x_{1}^{\prime}+u_{2}(x) x_{2}^{\prime}=0, \quad u_{1}^{\prime}\left(x^{\prime}\right) x_{1}+u_{2}^{\prime}\left(x^{\prime}\right) x_{2}=0, \\
u_{1}(x) u_{1}^{\prime}\left(x^{\prime}\right)+u_{2}(x) u_{2}^{\prime}\left(x^{\prime}\right)=0, \quad x_{1} x_{1}^{\prime}+x_{2} x_{2}^{\prime}=0, \\
v_{1}(x) x_{1}^{\prime}+v_{2}(x) x_{2}^{\prime}=0, \quad v_{3}(x) u_{1}^{\prime}\left(x^{\prime}\right)+v_{1}(x) u_{2}^{\prime}\left(x^{\prime}\right)=0, \\
v_{1}(x) v_{1}^{\prime}\left(x^{\prime}\right)+v_{2}(x) v_{2}^{\prime}\left(x^{\prime}\right)=0, \quad v_{3}(x) v_{3}^{\prime}\left(x^{\prime}\right)+v_{4}(x) v_{4}^{\prime}\left(x^{\prime}\right)=0, \\
w_{n}(x) w_{n}^{\prime}\left(x^{\prime}\right)+\bar{w}_{n}(x) \bar{w}_{n}^{\prime}\left(x^{\prime}\right)=0, \quad x_{1} x_{1}^{\prime}+x_{2} x_{2}^{\prime}=0,
\end{gathered}
$$

in which $u_{i}=0$ is a general conic, $v_{i}=0$ a conic through two fixed points, and $w_{n}=0$ a curve of order $n$ having $(0,0,1)$ for a point of multiplicity $n-2$.

All these forms are included in (5), which is a particular form of Type IX.

28. Fixed loci. Suppose a curve $c$ of one system meets a curve $k$ of another system in $s$ variable points, of which $s-2$ always lie on a fixed curve, leaving but two variable intersections. It can be shown that such cases can always be reduced to one or another of those previously considered, even when such fixed loci exist in both planes. 
29. Proof of non-existence of other types. It remains to be proved that any $(2,2)$ point correspondence between two planes can be reduced by birational transformation to one of the eleven types already obtained. The curves defined by (1) and (2) each belong to linear systems, any curve of one system having two variable intersections with the curves of the other system in the same plane. In the determination of pairs of systems of this kind in a plane $(x)$, it should be remembered, that by a birational transformation of the plane, and by taking as parameters functions which define the curves of a suitably chosen cremona net in a plane $(y)$ we can use any such pair to set up a $(1,2)$ correspondence between the planes $(x)$ and $(y)$, which is either a Geiser or Jonquières or Bertini correspondence, expressed in the normal form. We need therefore consider only such pairs of linear systems, as can be used to define one of these three correspondences. We therefore have (1) lines and conics, (2) conics and conics each with the same two basis points, (3) line pencil vertex $O$ and curves of order $n$, having $O(n-2)$ fold, (4) the image in $(x)$ of a pair of systems in $(y)$ having one variable intersection, namely either a Cremona net or the lines of the plane $(y)$, or a line pencil vertex $O$ and curves of order $n$ having $O(n-1)$-fold.

The first three cases give the Types $\mathrm{I}$ to $\mathrm{V}$, the fourth gives the Types VI to XI. The classification is therefore complete.

Cornell University November, 1916 